\title{
Benchmarking über die Fallnormkosten - bitte Gleiches mit Gleichem!
}

\author{
Mittels der Fallnormkosten sollen die Spitäler hinsichtlich ihrer Effizienz gebench- \\ markt werden. Die Universitätsspitäler begrüssen dies. Allerdings muss gewährleis- \\ tet sein, dass «Gleiches mit Gleichem» verglichen wird. Bei einem gemeinsamen \\ Benchmarking der Universitätsspitäler mit den nicht universitären Spitälern ist \\ diese Voraussetzung nicht gegeben.
}

Simon Spika, Hugo Keune

UniversitätsSpital Zürich, Direktion Finanzen
Korrespondenz:

Dr. oec. Simon Spika

UniversitätsSpital Zürich

Direktion Finanzen

Rämistrasse 100

CH-8091 Zürich

Tel. 0442559163

simon.spika[at]usz.ch

\section{Einleitung}

Mit Artikel 49 Abs. 1 KVG legt der Gesetzgeber fest, dass sich die Spitaltarife an der Entschädigung jener Spitäler orientieren sollen, «[...] welche die tarifierte obligatorisch versicherte Leistung in der notwendigen Qualität effizient und günstig erbringen». Geht man davon aus, dass der Gesetzgeber mit «effizient» der allgemeinen Definition von Effizienz folgt, so muss ein Spital A im Sinne von Art. 49 KVG effizienter als Spital B gelten, wenn es bei gleichem Input im Bereich der stationären, obligatorisch versicherten Leistungen einen grösseren Output produziert als Spital B es tut [1]. Dieser Vergleich setzt voraus, dass sich sowohl der Input als auch der Output beider Spitäler vergleichbar darstellen lassen. Während sich für den Input die Kosten sozusagen als «natürliche» gewicht - als Effizienzkennzahl gefestigt, anhand derer die Spitäler verglichen werden [4]. Zugegebenermassen liegt der Vorteil dieser Kennzahl darin, dass sie leicht zu ermitteln und prinzipiell leicht zu verstehen ist. Sie bedarf jedoch einer differenzierten Interpretation. Denn ein unkritischer Beobachter kommt schnell zum Ergebnis, dass Spital A tatsächlich effizienter ist als Spital B, wenn Spital A auf dem Papier die niedrigeren Fallnormkosten aufweist. Dabei würde der Beobachter aber implizit unterstellen, dass die Kostengewichte all die Unterschiede in der Leistungserbringung der Spitäler korrekt abbilden können und er Gleiches mit Gleichem vergleicht. Doch können die Kostengewichte, die im Grunde ja die Kostenrelation eines durchschnittlichen Spitals darstellen, diese Bedingung wirklich erfüllen? Kann

\section{«Universitätsspitäler erfüllen einen in der Spitallandschaft einzigartigen}

\author{
Aufgabenverbund aus Grundversorgung, Notfallabdeckung, Spitzen- \\ medizin sowie Forschung und Lehre.»
}

\begin{abstract}
Messgrösse anbieten, ist die Sachlage beim Output viel schwieriger. Denn die Spitäler sind untereinander sehr heterogen, in der Leistungsvielfalt wie auch in der Art und Weise, wie die Leistungen erbracht werden. Entsprechend anspruchsvoll ist es, den Output eines Spitals mit all seinen Facetten vergleichbar darzustellen. Davon zeugen nicht zuletzt die komplexen, multivariaten Ansätze in der wissenschaftlichen Literatur der Gesundheitsökonomik [1-3]. Trotzdem scheint die Frage in der Praxis beantwortet: In der aktuellen Tarifdiskussion wird gemeinhin davon ausgegangen, dass die Summe der SwissDRG Kostengewichte, also der Casemix, eine geeignete Messgrösse für den Output eines Spitals im stationären Bereich darstellt. Auf Basis dieser Annahme haben sich die sogenannten «Fallnormkosten» die anrechenbaren Kosten pro SwissDRG-Kosten-
\end{abstract}

sich der Beobachter wirklich darauf verlassen, dass Spital B nicht etwa nur deshalb höhere Fallnormkosten aufweist, weil es im Vergleich zu Spital A in besonderem Masse Leistungen erbringt, die von den Kostengewichten nicht dargestellt werden können, die aber im Sinne der obligatorischen Krankenversicherung sinnvoll, wenn nicht gar notwendig sind?

\section{Universitätsspitäler erbringen besondere Leistungen}

Für den Fall, dass ein Universitätsspital mit einem nicht universitären Zentrums- oder Regionalspital verglichen wird, lautet die Antwort klar: Nein. Denn die Universitätsspitäler erfüllen einen in der Spitallandschaft einzigartigen Aufgabenverbund aus Grundversorgung, Notfallabdeckung, Spitzenmedizin sowie Forschung und Lehre [5]. Aus diesem ein- 


\section{Abbildung 1}

Der Anteil der hochdefizitären Fälle ist bei den Universitätsspitälern (BfS Kategorie K111) deutlich höher als bei den Spitälern der restlichen Kategorien (linke Seite). Dieses Ungleichgewicht wird nicht durch die sogenannten «hochprofitablen» Fälle ausgeglichen (rechte Seite). Ein Fall wird hier als «hochdefizitär» bezeichnet, wenn bei einer Vergütung unter SwissDRG bei einer Baserate von 9764 CHF dem Spital ein grösserer Verlust als 30000 CHF entsteht. Entsprechend wird ein Fall, der dem Spital einem Gewinn von mehr als 30000 CHF erbringt, als «hochprofitabel» bezeichnet. Die Zahlen der Abbildung wurden im Rahmen einer Studie der Polynomics AG und des UniversitätsSpitals Zürich erstellt und basieren auf der BfS-Fallkostenstatistik des Jahres 2011 [6].

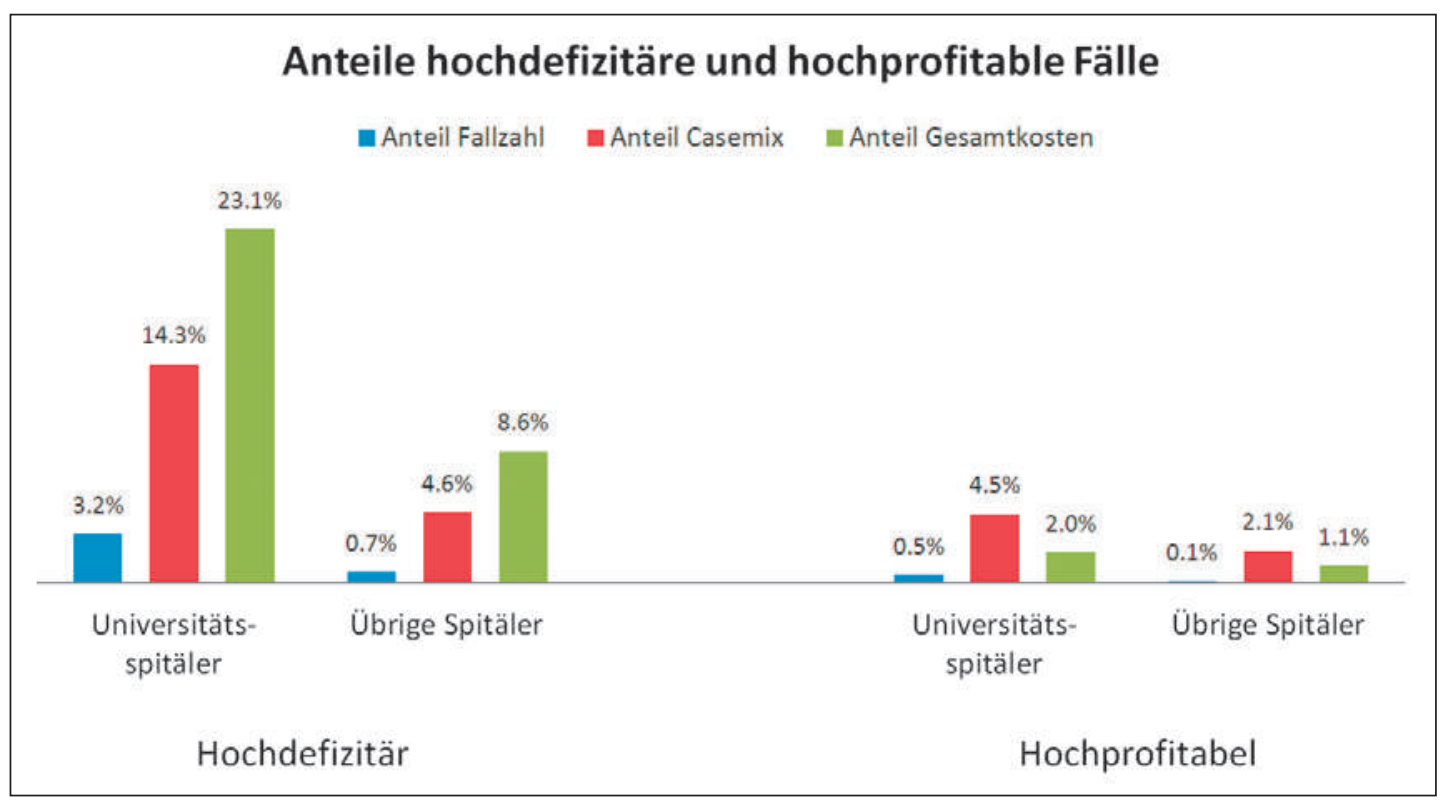

zigartigen Aufgabenverbund entstehen Möglichkeiten und Notwendigkeiten bei der Leistungserstellung, durch die sich die Universitätsspitäler auch im Bereich der obligatorisch versicherten Behandlung erheblich von den nicht universitären Spitälern unterscheiden. Doch diese Unterschiede können von den SwissDRG-Kostengewichten als durchschnittliche Kostenrelationen nicht ausreichend dargestellt werden. Hierzu zwei Beispiele:

\section{Beispiel «Unzureichende Abbildung der spezifischen Fallpopulation»}

Zum typischen Behandlungsspektrum der Universitätsspitäler als Maximalversorger gehört ein Anteil besonders schwerer Fälle und Fälle mit seltenen Erkrankungen. Bei diesen besonderen Fällen versagt
Da ihr «Beitrag» zum Leistungsausweis des Spitals, dem Casemix, viel zu gering ausfällt, entfalten diese Fälle eine grosse Hebelwirkung auf die Höhe der Fallnormkosten. Zwar lässt sich einwenden, dass diese Fälle auch bei den nichtuniversitären Spitälern vorkommen. Wie die linke Seite von Abbildung 1 zeigt, ist der Anteil dieser «hochdefizitären» Fälle in den Universitätsspitälern jedoch bedeutend höher. Das gilt im Übrigen auch noch dann, wenn man den Einfluss der «hochprofitablen» Fälle berücksichtigt, denen unter SwissDRG ein zu hohes Kostengewicht zugeordnet wird (rechte Seite von Abbildung 1).

\section{Beispiel «Integrale Interdisziplinarität»}

Neben dem Fallspektrum bestehen auch essentielle Unterschiede in den Behandlungsmöglichkeiten

\section{«Die Universitätsspitäler sehen ein exklusives Benchmarking unter den fünf Universitätsspitälern als momentan einzig praktikable Lösung.»}

die SwissDRG-Tarifstruktur jedoch häufig insofern, als dass sie diesen Fällen ein Kostengewicht zuweist, das angesichts der komplexen und teuren Behandlung zu niedrig ist, um den hohen Aufwand korrekt widerzuspiegeln. Wenngleich gering in der Zahl, so verursachen diese Fälle sehr hohe Kosten, so dass ihr Anteil an den Gesamtkosten des Spitals erheblich ist. und Behandlungsansätzen. Als Endversorger ist es die Aufgabe der Unispitäler, den Patienten, die darauf angewiesen sind, spitzenmedizinische Leistungen anzubieten, die auf der einen Seite mit entsprechendem Aufwand verbunden sind [7], sich auf der anderen Seite aber nicht ausreichend in den durchschnittlichen Kostenrelationen der SwissDRG-Tarif- 


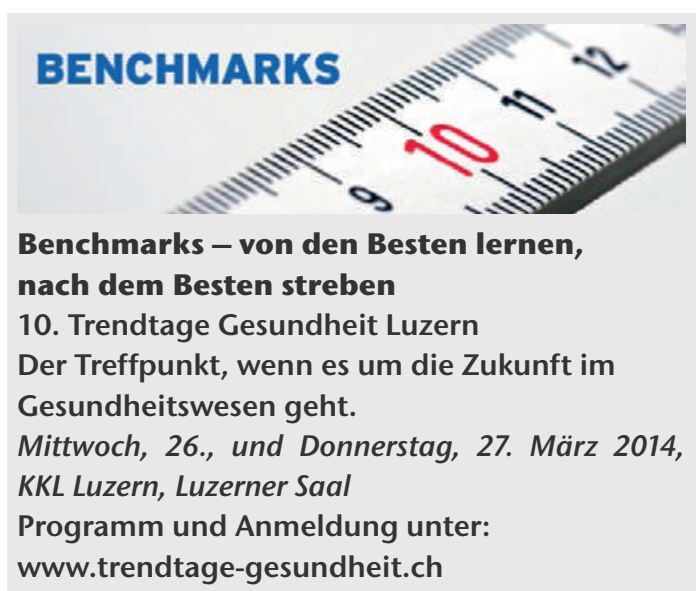

struktur widerspiegeln. Diese Leistungen zeichnen sich u.a. in besonderem Masse durch eine integrale interdisziplinäre Vernetzung der verschiedenen Fachbereiche aus. Hier sind etwa interdisziplinäre Konferenzen wie Tumorboards $\mathrm{zu}$ nennen, die in den Universitätsspitälern in einem viel bedeutenderen Masse institutionalisiert sind als in Zentrumsoder Regionalspitälern. Tumorboards bilden als effiziente Plattform des interdisziplinären Dialoges von Spezialisten der verschiedenen Fachabteilungen ein zentrales Element eines gemeinsam abgestimmten Vorgehens, das notwendig ist, um das bestmögliche Behandlungsergebnis für den Patienten zu erzielen. Die Interdisziplinarität wird aber nicht nur in planbaren Situationen gelebt. Sie ist auch dann von entscheidender Bedeutung, wenn in akuten Notfallsituationen das schnelle Eingreifen von Spezialisten verschiedener Fachdisziplinen erforderlich ist. An Universitätsspitälern wird eine rasche und koordinierte Zusammenarbeit der Spezialisten der verschiedenen Fachabteilungen 24 Stunden an 365 Tagen im Jahr auf höchstem medizinischem Niveau ermöglicht. Dies gilt insbesondere für die notfallmässige Behandlung schwierigster Fälle, die nicht selten von anderen Spitälern zugewiesen werden.

\section{Vergleichbarkeit gegeben? Nein!}

Dies sind nur zwei Beispiele für spezifische Leistungen der Universitätsspitäler, die zwar als anrechenbare Kosten die Fallnormkosten beeinflussen, gleichzeitig aber nicht über die Kostengewichte als Output ausgewiesen werden können. Die daraus resultierende Erhöhung der Fallnormkosten ist daher ent- schieden nicht als Ineffizienz $\mathrm{zu}$ interpretieren. Genau dies wäre aber bei einem undifferenzierten Benchmarking der Fall, was eine nicht gerechtfertigte Benachteiligung der Universitätsspitäler darstellen würde.

Gleichwohl stehen die Universitätsspitäler hinter SwissDRG, und es ist völlig klar, dass die unzureichende Abbildung der hier angesprochenen Leistungen nur zum Teil als Mangel der Tarifstruktur von SwissDRG zu qualifizieren ist. Ein System, das jegliche Unterschiede darstellen kann, wäre von unbeherrschbarer Komplexität. Es ist denn erklärtermassen auch längerfristig nicht das Ziel der SwissDRG AG, alle Kostenunterschiede der Leistungserbringung in der Tarifstruktur abzubilden. Ebenso befürworten die Universitätsspitäler das Benchmarking. Denn genau wie in allen anderen Spitälern wird auch in den Universitätsspitälern die Notwendigkeit einer effizienten Leistungserbringung erkannt, und genau wie die anderen Spitäler unternehmen sie umfangreiche Massnahmen zur Steigerung der Effizienz. Allerdings muss das Benchmarking differenziert erfolgen, um eine Benachteiligung der Universitätsspitäler zu vermeiden. Die Universitätsspitäler sehen deshalb ein exklusives Benchmarking unter den fünf Universitätsspitälern als momentan einzig praktikable Lösung, die beides gewährleistet: den Anreiz zur Steigerung der Effizienz sowie den Vergleich von Gleichem mit Gleichem.

\section{Literatur}

1 Hollingsworth, B. The Measurement of Efficiency and Productivity of Health Care Delivery. Health Economics. 2008;17(10):1107-28.

2 Burgess, JF. Productivity Analysis in Health Care, in The Elgar Companion to Health Economics. Cheltenham, Northampton: A.M. Jones; 2006. S.335-342.

3 Widmer PK, Zweifel P, Farsi M. Accounting for Heterogeneity in the Measurement of Hospital Performance. Working paper series, Department of Economics, University of Zurich; 2011 (No. 52).

4 Regierungsratsbeschluss Nr. 278 vom 13.03.2013: Kanton Zürich.

5 Fischer W. Universitätsmedizin und DRGs. Wolfertswil: ZIM-Verlag; 2013.

6 Bundesamt für Statistik: Fallkostenstatistik der Krankenhäuser 2008 bis 2011.

7 Albrecht M, Kroemer HK, Strehl R. Finanzierung der Universitätsmedizin: An der Grenze der Belastbarkeit. Deutsches Ärzteblatt. 2013;110(3). 